\title{
Evolving for Creativity: Maximizing Complexity in a Self-Organized Multi-Particle System
}

\author{
Heiko Hamann ${ }^{1}$, Thomas Schmickl ${ }^{1}$, and Karl Crailsheim ${ }^{1}$ \\ Artificial Life Lab of the Department of Zoology, Karl-Franzens University Graz, \\ Universitätsplatz 2, A-8010 Graz, Austria, \\ \{heiko.hamann, thomas.schmickl, karl.crailsheim\}@uni-graz.at
}

\begin{abstract}
We investigate an artificial self-organizing multi-particle (also multi-agent or swarm) system consisting of many (up to $10^{3}$ ) reactive, mobile agents. The agents' movements are governed by a few simple rules and interact indirectly via a pheromone field. The system generates a wide variety of complex patterns. For some parameter settings this system shows a notable property: seemingly never-ending, dynamic formation and reconfiguration of complex patterns. For other settings, however, the system degenerates and converges after a transient to patterns of low complexity. Therefore, we consider this model as an example of a class of self-organizing systems that show complex behavior mainly in the transient. In a first case study, we inspect the possibility of using a standard genetic algorithm to prolongate the transients. We present first promising results and investigate the evolved system.
\end{abstract}

\section{Introduction}

Self-organizing systems such as natural or artificial organisms and swarms often form complex patterns [1-3]. In nature, these systems are subject to natural selection, which evolves complex patterns by adapting simple behavioral rules followed by the agents. In our work, we model similar complex systems and adapt them by applying Evolutionary Computation. These systems are interpreted as nonlinear dynamic systems that converge to a fixed point or to periodic behavior. Some systems, however, show seemingly stable (i.e., fixed point or periodic behavior) complex patterns although relevant steady states do not exist. Such systems rely on quasi-stationary (or quasi-periodic) states that are induced by long transients. The state of a system is said to be transient in the time segment between the initialization of the system and before a steady state (e.g., a fixed point, a periodic behavior, or a chaotic attractor) is reached. Examples of such systems showing quasi-stationary behavior are models of ecological systems $[4,5]$ and many phenomena surrounding us in our everyday life such as ourselves [6]. Typically, the transient shows a much more complex and interesting behavior compared to the steady state, the system is actually slowly converging to.

\footnotetext{
${ }^{1}$ Supported by: EU-IST-FET project 'SYMBRION', no. 216342; EU-ICT project 'REPLICATOR', no. 216240.
} 


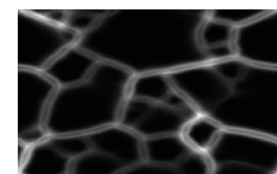

(a) $t=1 \times 10^{5}$.

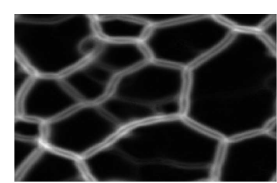

(b) $t=3 \times 10^{5}$.

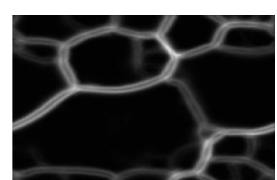

(c) $t=5 \times 10^{5}$.

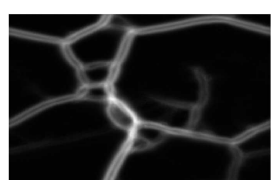

(d) $t=6 \times 10^{5}$.

Fig. 1. One pattern out of the variety of many of the investigated system $(N=3000$, $L=300, \eta=0.04$, parameters described below).

In this paper, we use a simple model of a multi-agent system. The agents move according to a few simple rules and interact indirectly via a (virtual) pheromone field. This model serves as an example of self-organized systems that depend on long transients. Based on only two parameters (resolution of the pheromone field discretization and/or the pheromone diffusion) we are able to change the behavior between relatively short transients (i.e., relatively fast convergence to a steady state of low complexity) and supposedly very long transients (actually so long that we even cannot be sure whether they are transient at all). We artificially restrict the system to rather low resolutions of the discretization leading to mostly manageable transients that are numerically simulated within a reasonable time. A first investigation of the transients was reported in [7]. Here, we try to prolongate the transients and to increase the complexity of the patterns by adjusting other parameters of the model. This is done by using a standard genetic algorithm [8]. Thus, we evolve parameter settings that result in long transients and high complexity which corresponds to increasing the time a self-organized system shows complex behavior.

We propose that Evolutionary Computation is a suitable tool to shape complex multi-particle systems in a desired manner. Together with the intrinsic creative potential of such particle systems (see Fig. 1), both techniques in combination serve as a powerful on-demand pattern generator. The objective of this work is, on the one hand, to produce a multi-particle system showing dynamic pattern formation, that might be applied in many ways, for example, in a novel robot controller concept or to generate pseudo-random textures for computer graphics (e.g., similar to Perlin's noise [9]). On the other hand, we want to determine the relevance of transients in self-organized systems and how to manipulate them.

\section{Mathematical Model}

In the following, we define the model of the investigated artificial multi-agent system as reported before in [7]. This system may also be called multi-particle system or swarm as it consists of many (up to $10^{3}$ ) reactive agents (automata that map receptions to actions without an internal world model). The model used here is based on and is very similar to the model of complex transport networks reported by Jeff Jones in [10]. The main differences between the models are a 
semi-continuous representation of agent data in Jones' model compared to a continuous representation in our model and a different density control (based on occupied patches in Jones' model, based on maximal pheromone field values here). Time is discrete in both models. Finally, both models are reduced to fully discrete models, because they are simulated on digital computers. However, the different methods of discretization do matter (rough discretization in patches and floating point arithmetic). The agents move in two-dimensional space with periodic boundary conditions (torus). The change of an agent's position $\mathbf{x}$ is defined by

$$
\frac{d \mathbf{x}}{d t}=\left(\begin{array}{c}
\cos \phi \\
\sin \phi
\end{array}\right) v,
$$

for a velocity set to a constant value $v>0$, except for the case that the local pheromone value is above a threshold $P_{\max }$ (then we set $v=0$ ). The change of the agent's direction $\phi$ is defined by

$$
\frac{d \phi}{d t}=\alpha\left(s_{l}(t), s_{c}(t), s_{r}(t)\right) \gamma(t)
$$

for $\alpha\left(s_{l}(t), s_{c}(t), s_{r}(t)\right) \in\{1,0,-1\}$ defining the direction of the turns (clockwise, no turn, or counterclockwise), $\gamma$ defining the absolute value of the turn angles, and for sensor values $s_{c}$ and $s_{l}$ that are defined by

$$
\begin{gathered}
s_{c}(t)=P\left(\begin{array}{l}
x_{1}+\cos (\phi) d \\
x_{2}+\sin (\phi) d
\end{array}\right), \\
s_{l}(t)=P\left(\begin{array}{l}
x_{1}+\cos (\phi-\psi) d \\
x_{2}+\sin (\phi-\psi) d
\end{array}\right),
\end{gathered}
$$

for a pheromone field $P$ representing the environment, $\mathbf{x}=\left(\begin{array}{l}x_{1} \\ x_{2}\end{array}\right)$, sensor angle $\psi$, and sensor distance $d . s_{r}$ is defined analog to $s_{l}$. Closely following [10], we define

$$
\alpha\left(s_{l}(t), s_{c}(t), s_{r}(t)\right)= \begin{cases}0, & \text { for } s_{c}(t)>s_{l}(t) \\ & \wedge s_{c}(t)>s_{r}(t) \text { (no turn) } \\ \pm 1, & \text { for } s_{c}(t)<s_{l}(t) \\ & \wedge s_{c}(t)<s_{r}(t) \text { (random turn) } \\ +1, & \text { for } s_{l}(t)<s_{r}(t) \text { (right turn) } \\ -1, & \text { for } s_{r}(t)<s_{l}(t) \text { (left turn) }\end{cases}
$$

whereas the order (from top to bottom) of the conditions matters. The random turn has a probability of $50 \%$ for +1 and $50 \%$ for -1 . We define

$$
\gamma(t)= \begin{cases}\phi_{\text {rot }}, & \text { for } t \in\{0, \tau, 2 \tau, \ldots\} \\ 0, & \text { else }\end{cases}
$$


for a constant rotation angle $\phi_{\text {rot }}$ and a time interval $\tau$ at which the agents turn and their directions are updated (in this work we set $\tau=1$ ). Thus, we obtain a synchronized system that is discrete in time. The system could, for example, be extended by defining $\gamma$ as a stochastic process. This would transform Eq. 2 into a stochastic differential equation.

The pheromone field $P$ is, in principle, defined by the standard diffusion equation

$$
\frac{\partial P(\mathbf{x}, t)}{\partial t}=D \nabla^{2} P(\mathbf{x}, t)-\eta P(\mathbf{x}, t)+\theta \sum_{i=1}^{N} \delta\left(\mathbf{x}-\mathbf{x}_{i}(t)\right),
$$

for diffusion $D$, evaporation rate $\eta$, addition $\theta$ (the Dirac delta indicates that an agent only contributes to the pheromone field at its position), number of agents $N$, and agent positions $\mathbf{x}_{i}(t)$. However, for simplicity and to reduce the computational complexity the diffusion and the evaporation are only executed at $t \in\{0,10 \tau, 20 \tau, \ldots\}$ unlike the addition process that is executed at $t \in$ $\{0, \tau, 2 \tau, \ldots\}$.

As discussed above, the system is simulated on a digital computer. Thus, the pheromone field needs to be discretized. This is done by a grid. Here, the grid is chosen to be always quadratic and the number of grid points is given by $L^{2}$. In our implementation, the diffusion is not normalized in correspondence to the resolution of the grid (as it would be necessary in classical numerics).

If provided with a sufficient resolution of the grid, that is discretizing the pheromone field, the model shows a huge variety of complex patterns [7]. These patterns form, collapse partially, form again, and seem never to recur. This way the system shows high creativity that is very different compared to many other self-organizing systems that converge (quickly) to a steady state. This is related to the concept of synergetics where modes of high dynamics are governed by modes of slow dynamics [11]. Throughout this work, the agents' positions are initialized by a random uniform distribution throughout the whole space. See Fig. 1 for some examples of patterns formed in the pheromone field and see Table 1 for the parameters used, if not stated explicitly.

\section{Complexity and Transients}

In order to investigate the model concerning transients we need a method determining when a steady state is reached. In a high-dimensional system this is, generally, difficult. In addition, we want also to evolve complex and dynamic patterns. Thus, we restrict ourselves to a simpler (also concerning computational complexity) but effective method. As we know from experience with the simulator, the steady states are all of low complexity (patterns formed out of straight lines with two, one, or no bifurcations or only clusters). Instead of checking for a steady state it would suffice to find a metric that measures the complexity of the dynamics.

In this work, we use the following metric: We put a second grid (called counting grid) of lower resolution (here: $30 \times 30$ ) over the pheromone grid and count 
Table 1. Standard parameters.

\begin{tabular}{|l|c|}
\hline \begin{tabular}{l|c} 
sensor angle $\psi$ \\
rotation angle $\phi_{\text {rot }}$
\end{tabular} & $45^{\circ}$ \\
rotation period $\tau$ & 1 [time units] \\
surface area of the torus $s^{2}$ & $1 \times 1[\text { length units }]^{2}$ \\
grid length $L$ & 150 \\
grid resolution & $L / s=150[1 /$ length units] \\
sensor distance $d$ & $0.035[$ length units] \\
velocity $v$ & $0.01[$ length units $/ \tau]$ \\
diffusion $D$ & $0.1[(1 / L) /(10 \tau)]$ \\
evaporation $\eta$ & $0.04[1 /(10 \tau)]$ \\
addition $\theta$ & $5[1 / \tau]$ \\
active cell threshold $\delta_{\text {active }}$ & 30 \\
max. pheromone value $P_{\max }$ & 300 \\
simulated steps & $5 \times 10^{4}[$ time units] \\
\hline
\end{tabular}

the grid points of the pheromone grid that are above a threshold $\delta_{\text {active }}$ (called active cells). If there are more than $50 \%$ active cells within one cell of the counting grid we mark it as 'on' (otherwise 'off'). It is sufficient to calculate the current state of the counting grid only every 200 time steps because the motion of the agents and the pattern dynamics occur on different time scales. Finally, the value of our complexity metric $\beta$ is determined by counting the differences between the current counting grid and the one obtained 200 time steps before (thus, $\beta \in\left\{0, \ldots, 30^{2}\right\}$ ). See Fig. 2 for examples of the evolution of the complexity measure $\beta$ for different parameter settings. Fig. 2(a) shows an example of a pattern that degenerates quickly to a collection of stationary clusters. Fig. 2(b) shows a pattern that degenerates to a traveling pattern (causing oscillations in $\beta$ as it moves over the counting grid) of three lines and two bifurcations after a transient of about 66,000 time steps.

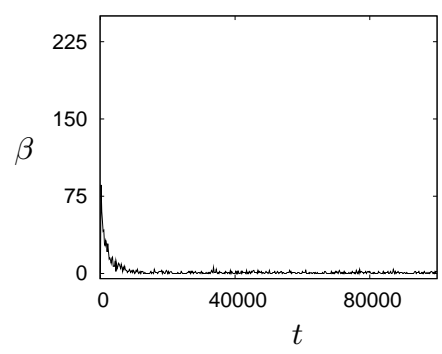

(a) $\psi=60^{\circ}, \phi_{\text {rot }}=60^{\circ}$.

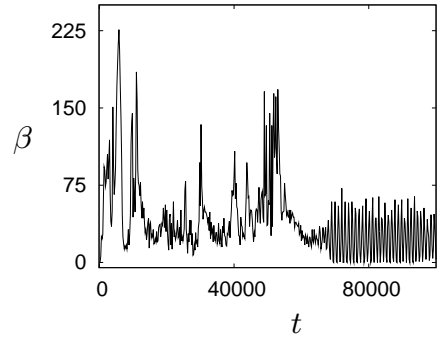

(b) $\psi=30^{\circ}, \phi_{\text {rot }}=30^{\circ}$.

Fig. 2. Examples of the temporal evolution of the complexity measure $\beta(v=0.01$, $d=0.06, \eta=0.08, D=0.05, N=500)$. 


\section{Evolving Complex and Long Transients}

In this section, our new approach of evolving parameter sets that generate long transients with complex patterns is reported. The used optimizer is a standard genetic algorithm based on GALOPPS 3.2.4 by Erik Goodman [12]. Four parameters were varied within given intervals: rotation angle $\phi_{\text {rot }} \in\left[10^{\circ}, 90^{\circ}\right]$, sensor angle $\psi \in\left[10^{\circ}, 90^{\circ}\right]$, diffusion constant $D \in\left[10^{-3}, 10^{-1}\right]$, and number of agents $N \in\left[2^{0}, 2^{10}\right]$. The velocity of the particles was fixed because changes in the velocity are balanced by the diffusion constant as only the relation between these two parameters matters. The restriction to these intervals does only exclude parameter settings leading to irrelevant patterns (based on our experience). The values were encoded in a 10 bit Gray code. Mutations were single bit flips. Recombinations were two-point crossovers with leaving the 10-bit-groups intact. The selection mechanism was stochastic universal sampling. The population size was set to 20 , the probability of a recombination was set to 0.05 , and the probability of a mutation was set to 0.05. The fitness was defined as the sum of all changes in the counting grid until less than ten changes occurred for ten successive checks (i.e., 2,000 time steps). The simulation was stopped after $10^{5}$ time steps in any case. For the used random uniform initialization of agent positions and directions this value was stochastic. The fitness was the mean of three simulation runs of the individual. A single run of 500 generations takes about three days of computing time on a contemporary desktop computer (single core). The averaged results of four runs of the genetic algorithm are shown in Fig. 3(a).

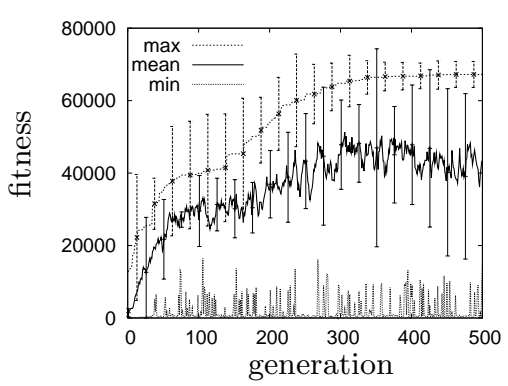

(a) Fitness over 500 generations.

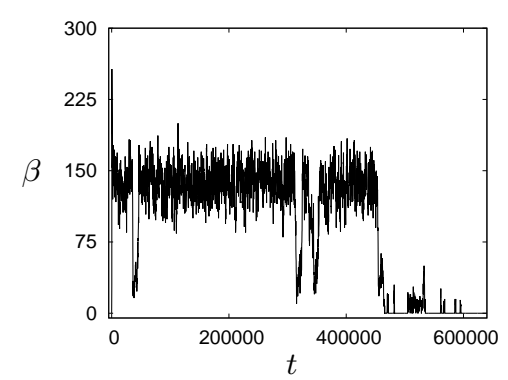

(b) Complexity measure over time.

Fig. 3. Left: max., mean, and min. fitness of four runs over 500 generations (errorbars indicate $95 \%$ confidence intervals). Right: Temporal evolution of the complexity measure $\beta$ of the best individual found.

Beginning with a mean fitness of 1,446, a peak mean fitness of 51,293 was reached after 306 generations. The mean fitness of the best individual after 500 generations was 67,393 . The best individuals of the four runs were very 
similar; we give the averages and the standard deviations: $\phi_{\text {rot }}=89.4^{\circ} \pm 0.39$, $\psi=10.1^{\circ} \pm 0.16, D=0.00125 \pm 5 \times 10^{-4}$, and $N=582.5 \pm 38.2$.

The following analysis of the best individual shows the high degree of adaptivity to the fitness function. The evolution of the complexity measure for an example run is shown in Fig. 3(b). The dynamics in the counting grid were very high (cf. Fig. 2) with few interruptions. After about 450,000 time steps the pattern degenerates to a single line in this example (typically the observed transient was even longer). Thus, the pattern survives for the whole evaluation period $\left(10^{5}\right.$ steps). The pheromone field of characteristic phases is plotted in Fig. 4.

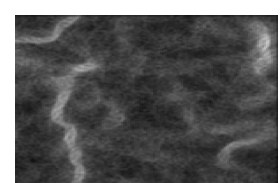

(a) Chaotic phase.

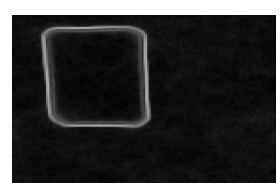

(b) Expanding rect.

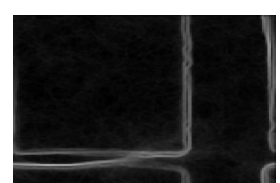

(c) Colliding rect.

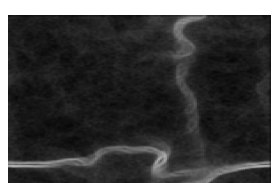

(d) Chaotic phase.

Fig. 4. Pheromone field of best individual.

The system shows, for most of the time, a rather chaotic behavior, see Fig. 4(a). However, about two times per 300,000 time steps it forms an expanding rectangle, see Fig. 4(b) to 4(d). The agents deform this rectangle because of the rotation angle $\phi_{\text {rot }}=89.609^{\circ} \neq 90^{\circ}$. Through a non-trivial process this leads to an expansion which continues until the rectangle collides with itself (space is toroidal). Then another chaotic phases begins. These rectangle-formation-phases are clearly identified in Fig. 3(b) as the short phases of low values $(10<\beta<70)$. The high fitness of this individual does not depend on the toroidal form of space as observed in runs of the genetic algorithm with strict bounds at all four sides (non-toroidal, data not shown).

\section{Conclusions and Outlook}

In this paper, we applied genetic algorithms successfully to increase the creativity and complexity of self-organizing systems by prolongating the transient. We investigated a multi-agent system with simple rules that shows a huge variety of complex patterns. This model is interpreted as an example of self-organized systems that rely on long transients because the investigated systems only show complex behavior before the steady state is reached. Parameter settings were evolved that lead to longer transients and, thus, maximize the time complex behavior is observed. The presented model is a microscopic (bottom-up) model inspired by slime molds [10]. The observed patterns bear similarities to macroscopic (top-down) differential equation models of slime molds as reported in [13]. Other relevant models showing similar patterns are the macroscopic reactiondiffusion models of animal coats $[14,15]$. In our microscopic model changes in 
the parameters result in a huge variety of patterns. Hence, small changes of parameters might result in big changes in the quality of the patterns. Thus, difficulties in evolving patterns with desired properties would be expected. In this paper, we have shaped the emergent properties of a self-organizing system according to our requirements by evolution. The fitness landscape seemed to be good-natured and rather smooth because all four runs were successful and resulted in very similar individuals. In the future, we will further explore the abilities of multi-particle systems in combination with Evolutionary Computation. We will investigate those parameters in detail, which produce constantly changing, complex patterns. Our goal is to shape these patterns in a way that we are either able to manually construct them or to evolve them in a desired way (e.g., to connect predefined points in the modeled space or to obtain inhomogeneous textures). Possible applications for such dynamic pattern generators are ranging from artificial life, swarm robotics, and collective intelligence to arts and other forms of visual design and computer graphics.

\section{References}

1. Thompson, D.W.: On Growth and Form: The Complete Revised Edition. Dover Publications (1992)

2. Murray, J.D.: On the mechanochemical theory of biological pattern formation with application to vasculogenesis. Comptes Rendus Biologies 326(2) (2003) 239-252

3. Bonabeau, E., Dorigo, M., Theraulaz, G.: Swarm Intelligence: From Natural to Artificial Systems. Oxford Univ. Press (1999)

4. Hastings, A.: Transient dynamics and persistence of ecological systems. Ecology Letters 4 (2001) 215-220

5. Hastings, A.: Transients: the key to long-term ecological understanding? TRENDS in Ecology and Evolution 19(1) (January 2004) 39-45

6. Prigogine, I.: The End of Certainty. Free Press (1997)

7. Hamann, H.: Pattern formation as a transient phenomenon in the nonlinear dynamics of a multi-agent system. In: MATHMOD 2009 - 6th Vienna International Conference on Mathematical Modelling. (2009)

8. Holland, J.H.: Adaptation in Natural and Artificial Systems. Univ. Michigan Press, Ann Arbor, MI (1975)

9. Perlin, K.: Improving noise. ACM Transactions on Graphics, Special issue: Proceedings of ACM SIGGRAPH 2002 21(3) (2002) 681-682

10. Jones, J.: The emergence and dynamical evolution of complex transport networks from simple low-level behaviours. J. of Unconv. Comp. (2009) accepted.

11. Haken, H.: Synergetics - an introduction. Springer-Verlag, Berlin, Germany (1977)

12. Goodman, E.D.: An introduction to galopps-the genetic algorithm optimized for portability and parallelism system, release 3.2. Technical Report 96-07-01, Intelligent Systems Laboratory and Case Center for Computer-Aided Engineering and Manufacturing, Michigan State University (1996)

13. Höfer, T., Sherratt, J.A., Maini, P.K.: Dictyostelium discoideum: Cellular selforganisation in an excitable medium. Proceedings of the Royal Society London B 259(1356) (1995) 249-257

14. Murray, J.D.: A prepattern formation mechanism for animal coat markings. J. Theor. Biol. 88 (1981) 161-199

15. Meinhardt, H.: Models of biological pattern formation. Academic Pr., NY (1982) 